\title{
Evaluation of a new tension relief system for securing wound closure: A single-centre, Chinese cohort study
}

\author{
Zhang Huahui $\mathrm{MD}^{1^{*}}$, Xue Dan $M D \mathrm{PhD}^{1 *}$, Jiang Hongfei $\mathrm{MD}^{2}$, Hu Hang $M D \mathrm{PhD}^{3 *}$, Han Chunmao $\mathrm{MD}^{3}$, \\ Ren Haitao MD $\mathrm{PhD}^{3}$, Yu Jianxin MD PhD ${ }^{1}$, Tao Zhiping MD PhD ${ }^{1}$
}

Z Huahui, X Dan, J Hongfei, et al. Evaluation of a new tension relief system for securing wound closure: A single-centre, Chinese cohort study. Plast Surg 2016;24(3):177-182.

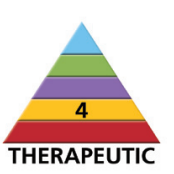

BACKGROUND: Wounds that have been closed under excessive tension, and skin defects that cannot be closed primarily, pose a daily challenge for the reconstructive surgeon.

OBJECTIVE: To evaluate a new tension relief system (TRS) device for skin stretching and secure wound closure.

METHODS: From September 2013 to March 2014, a consecutive series of 41 Chinese patients with 43 wounds were enrolled for application of 50 cycles of TRS therapy. TRS was used for two main clinical applications: closure of a variety of surgical/traumatic wounds; and securing wound closure after high-tension suture closure. Basic information and details regarding this therapy and its complications were recorded. Follow-up visits were conducted three to six months after wound closure.

RESULTS: Mean residual wound width decreased approximately $20 \%$ every two days during cycles of TRS therapy. Infection was the most common complication (five cases). Other complications included dehiscence (two cases) and pressure ulcer (one case). At the six-month follow-up visit, (21 wounds in 20 patients), both the extent of healing and the scar were acceptable.

DISCUSSION: There are no absolute contraindications to TRS therapy. The authors have formulated instructions for the prevention and treatment of the most common complications.

CONCLUSIONS: The results demonstrate that TRS therapy is a simple, effective method for primary closure of difficult wounds, and large skin and soft-tissue defects. Larger randomized studies are required to further evaluate of the effectiveness, indications, complications and cost effectiveness of this innovative TRS therapy.

Key Words: External skin expansion; Mechanical creep; Skin stretching; Skin stress relaxation; Wound closure

W ounds that have been closed under excessive tension or skin defects that cannot be primarily closed, pose a daily challenge to the reconstructive surgeon. Two major techniques have been developed for solving such problems: skin and/or soft-tissue flap or graft; and skin and/or soft tissue elongation and expansion. Advantages of the expansion techniques include appropriate colour match, texture, sensibility, and substantial adnexa for aesthetic restoration of the resulting defect (1). However, conventional tissue expansion has been associated with a high complication rate, ranging as high as $40 \%$ to $50 \%$ (2).

In 2012, Topaz et al (3) first reported their clinical experience with a new tension relief system (TRS): a novel, simple, skin-stretching and secure closure system designed to harness both mechanical creep and stress relaxation principles. These investigators shared their

\section{L'évaluation d'un nouveau système de soulagement des tensions pour assurer la fermeture des plaies : une étude de cohorte monocentrique chinoise}

\begin{abstract}
HISTORIQUE : Les plaies fermées sous tension excessive et les anomalies cutanées qui ne peuvent pas être fermées par une intervention primaire représentent un problème quotidien pour le plasticien.

OBJECTIF : Évaluer un nouveau système de soulagement des tensions (SST) pour étirer la peau et assurer la fermeture des plaies.

MÉTHODOLOGIE : De septembre 2013 à mars 2014, une série consécutive de 41 patients chinois présentant 43 plaies ont été inscrits pour recevoir 50 cycles de traitement par SST. Le SST était utilisé pour deux principales applications cliniques : fermer diverses plaies chirurgicales ou traumatiques et assurer la fermeture des plaies après une suture à haute tension. Les chercheurs ont colligé l'information générale et détaillée au sujet de ce traitement et de ses complications. Les rendez-vous de suivi ont eu lieu de trois à six mois après la fermeture des plaies.
\end{abstract}

RÉSULTATS : La largeur des plaies résiduelles moyennes diminuait d'environ $20 \%$ tous les deux jours pendant les cycles de traitement par SST. L'infection était la complication la plus courante (cinq cas). Les autres complications étaient la déhiscence (deux cas) et l'ulcère de pression (un cas). Au rendez-vous de suivi six mois plus tard ( 21 plaies chez 20 patients), tant le processus de cicatrisation que la cicatrice étaient acceptables.

EXPOSÉ : Il n'y a pas de contre-indications absolues au traitement par SST. Les auteurs ont formulé des directives pour prévenir et traiter les complications les plus courantes.

CONCLUSIONS : Les résultats démontrent que le traitement par SST est une méthode simple et efficace pour assurer la fermeture primaire de plaies complexes et d'importantes anomalies de la peau et des tissus mous. Des études aléatoires plus vastes s'imposent pour approfondir l'évaluation de l'efficacité, des indications, des complications et du rapport coût-efficacité de ce traitement novateur par SST.

*Authors who contributed equally

${ }^{1}$ Department of Plastic Surgery, Second Affiliated Hospital, College of Medicine; ${ }^{2}$ Assisted Reproduction Unit, Department of Obstetrics and Gynecology, Sir Run Run Shaw Hospital, College of Medicine; ${ }^{3}$ Department of Burns and Wound Center, Second Affiliated Hospital, College of Medicine, Zhejiang University, Hang Zhou, People's Republic of China

Correspondence: Dr Hu Hang, Department of Burns and Wound Center, Second Affiliated Hospital College of Medicine, Zhejiang University, \#88 JieFang Road, Hang Zhou, Zhejiang Province, 310009, People's Republic of China. Telephone 0086-571-87783661,

fax 0086-571-87784585, e-mail huhangbob@aliyun.com 


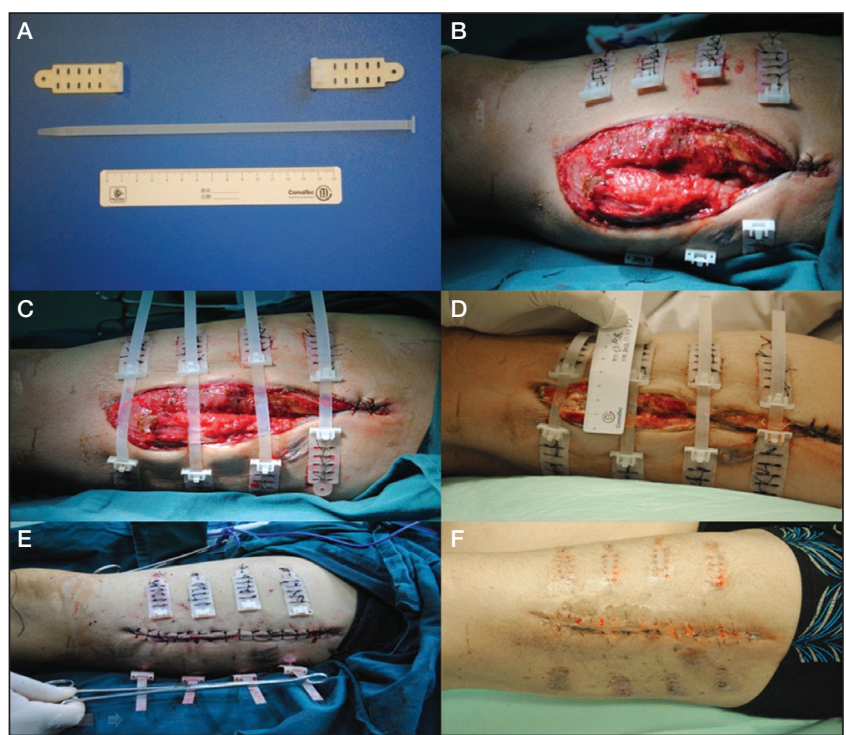

Figure 1) Surgical steps involved in tension relief system therapy. A The TopClosure $^{\circledR}$ (IVT Medical Ltd, Israel) device shown with attachment plates (APs) and approximation straps (AS). B APs deployed after complete debridement. C APs connected with AS, which are vertical to the wound/suture line. D Gradual stretching of skin by adjusting the TopClosure ${ }^{\circledR}$ device and measuring residual wound width during dress changing. E Wound closed by direct suture and TopClosure ${ }^{\circledR}$ device were kept for stress relaxing and protect the suture. F Wound completely healed, and both the suture and TopClosure ${ }^{\circledR}$ device removed

for two main clinical applications: closure of a variety of surgical/ traumatic wounds; and securing wounds that were closed under excessive tension. Exclusion criteria were malignant ulcers, heavily infected wounds, wounds with necrotic tissue that were not completely debrided, and damaged skin (eg, severe edema, skin-grafted areas and deeply scarred wound edges).

\section{Medical history and wound information}

Patient medical history and demographic information, including age, sex, smoking index, wound-related comorbidities (diabetes, varicose veins, paraplegia, anemia) and body mass index (BMI), was recorded. After each patient was enrolled, the patient's wound was carefully inspected, and information, including wound location, width, length, presence of exposed bone or internal fixation material, cause of injury, quality of the wound edge, skin and granulation tissue, and the presence of external fixator impeding skin closure, was recorded. Wound biopsy for tissue culture was performed to evaluate wound infection.

\section{Surgical technique and TRS application}

The surgical approach was designed to create a suitable environment for skin stretching. For wounds with severe infection and/or residual necrotic tissue, complete debridement of necrotic tissue was indispensable. The application of the TRS device was previously described by Topaz et al (3). The TRS set consists of two attachment plates (APs) that are interconnected by a long, flexible approximation strap (AS) (Figure 1A). Pairs of attachment plates were attached to the skin $1.5 \mathrm{~cm}$ to $2 \mathrm{~cm}$ from the wound edges, and were secured by a few sutures. The number of Top Closure ${ }^{\circledR}$ (IVT Medical, Ltd, Israel) TRS sets (one to four) required depended on the length of the wound and the anticipated tension associated with wound closure. The APs were attached to intact skin and were then connected to the AS, with vectors perpendicular to the wound edges (Figures 1B and 1C).

Wound dressing and skin stretching

Vacuum sealing devices (VSDs) (similar to the VAC device in Western countries) and functional silver dressing (Aquacell Ag [Convatec,
TABLE 1

Baseline characteristics

\begin{tabular}{lc}
\hline Characteristic & \\
\hline Male sex, $\mathrm{n}(\%)$ & $41(21)$ \\
Age, years & $38.06 \pm 2.91$ \\
Body mass index, $\mathrm{kg} / \mathrm{m}^{2}$ & $23.34 \pm 0.48$ \\
Smoking index (cigarettes/day $\times$ years) & $258.50 \pm 64.83$ \\
Diabetes, $\mathrm{n}$ & 3 \\
\hline
\end{tabular}

Data presented as mean $\pm S D$ unless otherwise indicated

\section{TABLE 2}

Wound information $(n=43)$

\begin{tabular}{lc}
\hline Wound etiology, $\mathrm{n}$ & 15 \\
Traffic accident & 5 \\
Large flap donor site & \\
Incision and decompression & 7 \\
of Compartment syndrome & \\
$\quad$ Incision and drainage of & 5 \\
Bone/fixation material chronic infection & 11 \\
$\quad$ Other injury (fall, crush, cuts) & \\
Wound location, $\mathrm{n}$ & 13 \\
Foot & 10 \\
Calf & 6 \\
Thigh & 6 \\
Forearm & 5 \\
Abdomen & 3 \\
Knee & $4.61 \pm 0.71$ \\
Original width, cm, mean $\pm \mathrm{SD}$ & $11.43 \pm 1.81$ \\
Original length, cm, mean $\pm \mathrm{SD}$ & 16 \\
External fixator, $\mathrm{n}$ & 30 \\
Bone/fixation material exposure, $\mathrm{n}$ & $18 / 13 / 12$ \\
Original wound management (VSD/Ag dressing/ \\
conventional dressing), $\mathrm{n}$ \\
Infection (microorganism biopsy result), $\mathrm{n}$
\end{tabular}

VSD Vacuum sealing device

USA] and SeaSorb Ag [Coloplast, USA]) were applied during wound closure. For larger wounds, those sufficiently wide and deep for VSD materials, vacuum sealing is preferred. For smaller wounds that are too narrow and superficial to contain VSD material, functional silver dressing was applied. The first wound dressing change was performed three days after surgery and, for VSD-dressed wounds, only the gauze outside the VSD dressing was changed. Subsequently, wound dressing was changed every two days. Residual wound width (calculated from the residual distance between APs) was measured and the skin was stretched gradually by adjusting the TRS device (Figure 1D). For patients with VSD dressing, debridement surgery was repeated every seven days. The VSD dressing was replaced by a smaller dressing, and the APs were repositioned for further skin stretching.

\section{Wound closure and follow-up}

Once the wound was firmly approximated by the TRS device or a sufficient amount of relaxed skin was contained in both sides of VSD dressing, final surgery was performed. Another microorganism biopsy was obtained, and the wound was cleaned and closed using sutures. In several cases, when the tension of the suture line remained too high, the TRS device was also repositioned for relief of skin stress and to protect the sutures (Figure 1E). Conventional dressing change was performed every two days until the wound healed completely, and both the suture and the TRS device were removed (Figure 1F). A one-week follow-up was scheduled to record possible short-term complications. A follow-up visit was scheduled six months after healing to assess and photograph the scars. 
TABLE 3

Details regarding TopClosure ${ }^{\circledR}$ (IVT Medical, Israel) tension relief system (TRS) therapies

\begin{tabular}{lc}
\hline TopClosure TRS system, applied sets, $\mathrm{n}$ & 130 \\
Pairs per wound, sets & $2.59 \pm 0.22$ \\
Wound length per set, cm & $4.40 \pm 1.52$ \\
Duration of each cycle, days & $16.72 \pm 4.48$ \\
\hline
\end{tabular}

Data presented as mean $\pm S D$ unless otherwise indicated

\section{Recording and management of complications}

During TRS therapy, complications, such as local or systemic infection, pressure ulcers beneath the APs and wound dehiscence, were recorded and targeted treatments performed as necessary. For patients with infection, systemic antibioticss were administered according to microorganism sensitivities. For pressure ulcers, a cardboard mat was placed beneath the APs to reduce the pressure or, if necessary, the APs were removed. For wound dehiscence, a conventional dressing change was performed and the APs were repositioned to promote wound healing.

\section{Data collection and statistical analysis}

Continuous variables are expressed as mean $\pm \mathrm{SD}$. Enumeration data are expressed as percentages; $P<0.05$ was considered to be statistically significant.

The original wound width ranged from $1 \mathrm{~cm}$ to $10 \mathrm{~cm}$. For statistical analysis, the residual wound width $(\mathrm{Rw})$ during TRS therapy was standardized as $\mathrm{Rw} \%$ :

$$
\begin{aligned}
& \text { Rw\% } \% \text { residual wound width }(\mathrm{cm}) / \text { original wound width }(\mathrm{cm}) \\
& \qquad \times 100 \% .
\end{aligned}
$$

Because the VSD dressing is not compressible, and the decrease of the wound width along the wound edge was not equal, $\mathrm{Rw}$ was calculated as:

$$
\begin{gathered}
\mathrm{Rw}=\text { original } \text { wound width }- \text { (original distance between } \\
\text { APS }- \text { residual distances between APS }) .
\end{gathered}
$$

Complications were recorded as binary data (ie, $0 / 1$ ). For patients with infection, the microorganism biopsy result and clinically symptomatic infections were recorded as descriptive data. For variances between groups and different time points, independent sample $t$ tests or nonparametric independent sample tests were performed, depending on variable distribution. Statistical analysis was performed using SPSS version 11.5 (IBM Corporation, USA) for Windows (Microsoft Corporation, USA).

\section{Baseline characteristics}

\section{RESULTS}

A group of 41 patients ( 20 female and 21 male) was enrolled during the present investigation. Three patients had been diagnosed with diabetes mellitus. Demographic information, including sex, age and BMI, is summarized in Table 1.

\section{Wound information}

Forty-three wounds were investigated in 41 patients. These wounds had multiple causes including traffic accident, surgery, and incision and drainage of infected bone, or internal fixation materials. The original wound width ranged from $1 \mathrm{~cm}$ to $10 \mathrm{~cm}$. Details of these wounds are presented in Table 2 .

\section{TRS therapy}

A total of 50 TRS treatment cycles were applied to these 43 wounds. Forty-one TRS cycles were applied before suturing for gradual closure of 41 wounds in 39 patients; nine TRS cycles were applied following suturing to secure closure of high-tension wounds (Figure 2). A total

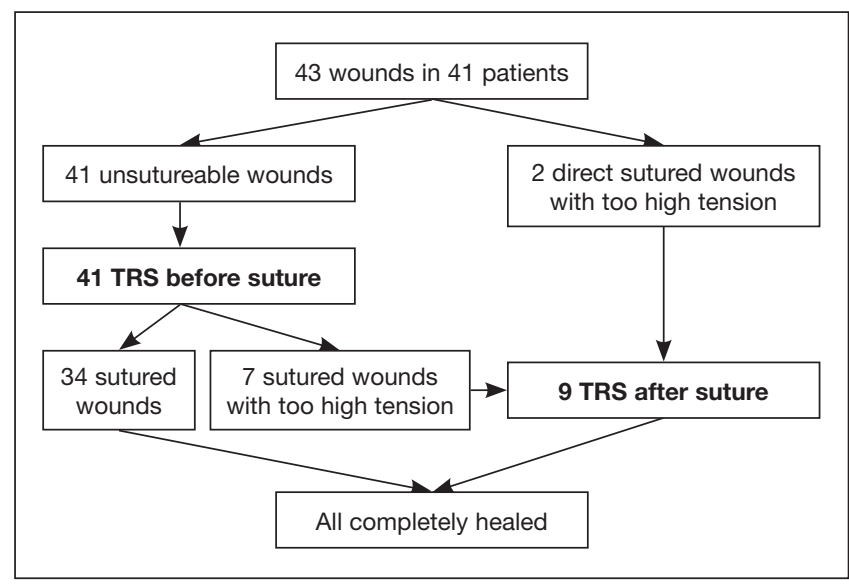

Figure 2) Therapeutic pathway of the study. TRS Tension relief system

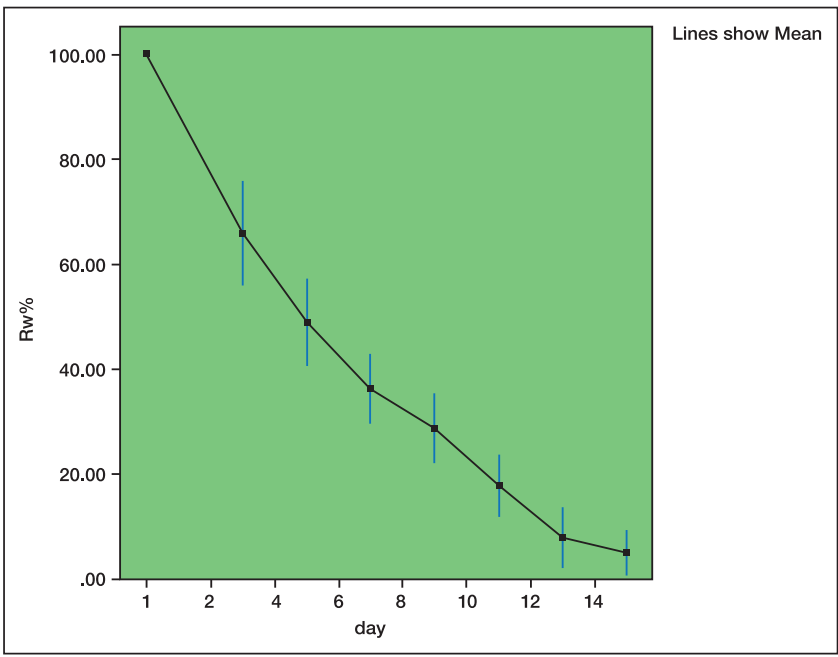

Figure 3) Decreasing curve of residual wound width (Rw\%) during cycles closing wounds

of 130 sets of the TRS system were applied (mean [ \pm SD] $2.59 \pm 0.22$ sets per wound). The number of sets of the TRS system on each wound was strongly correlated with wound length $(\mathrm{P}<0.05)$ : on average, one set per $4.40 \pm 1.52 \mathrm{~cm}$. The mean duration of the TRS treatment cycle was $16.72 \pm 4.48$ days.

For the 41 wounds treated with staged cycles of wound closure, the mean $\mathrm{Rw}$ decreased approximately $20 \%$ every two days during cycles of wound closure. One-way ANOVA tests demonstrated a significant difference between every two visit points. Details of TRS therapies are shown in Table 3. The decreasing trend of Rw\% is also presented in Figure 3.

\section{Wound management during TRS therapies}

During cycles for wound closures, VSD devices were applied on 15 wounds, while Ag dressing was performed on the other 28. During cycles to secure closed wounds, VSD devices were applied on two suture lines, while Ag dressing was applied to the other seven suture lines.

Final outcomes and complications during and after TRS therapies Forty-one wounds in 39 patients were successfully closed by sutures after one cycle of TRS wound closure. Subsequently, seven wounds underwent a further TRS cycle to secure sutured wounds; two wounds in the other two patients were directly sutured and had only one TRS cycle applied to secure wound closure. All of these wounds were completely healed at the one-week follow-up visit. At the three- and 


\section{TABLE 4}

Complication details during TopClosure ${ }^{\circ}$ (IVT Medical Ltd, Israel) tension relief system therapies

\begin{tabular}{llllll}
\hline Case & Original infection & Dressing & Final result & Symptoms & Time of occurrence \\
\hline 1 & Negative & VSD & Candida albicans & No & NA \\
2 & Enterobacter cloacae & $\mathrm{Ag}$ & Staphylococcus aureus & Yes & Suture (granulation resected) \\
3 & Enterobacter cloacae & $\mathrm{Ag}$ & Negative & No & NA \\
4 & Enterococcus faecalis + MRSA & $\mathrm{Ag}$ & MRSA & Yes & Suture (granulation resected) \\
5 & MRSE & $\mathrm{Ag}$ & Negative & No & NA \\
6 & Pseudomonas aeruginosa + MRSA & VSD & Pseudomonas aeruginosa + MRSA & Yes & Change VSD \\
7 & Pseudomonas aeruginosa + MRSA & VSD & Negative & No & NA \\
8 & MRSA & Ag & MRSE & Yes & Suture (granulation resected) \\
9 & Serratia marcescens & Ag & MRSA & Yes & Suture (granulation resected) \\
Wound dehiscence, $\mathrm{n}(\%)$ & & $2(4.65)$ healed after dress changing & \\
Pressure ulcer, $\mathrm{n}(\%)$ & $1(2.33)$ healed after dress changing & \\
Pain and itching, $\mathrm{n}(\%)$ & & $15(36.58)$ &
\end{tabular}

Ag Silver; MRSA Methicillin-resistant Staphylococcus aureus; MRSE Methicillin-resistant Staphylococcus epidermidis; NA Not applicable; VAD Vacumm sealing device

six-month follow-up visits, all wounds were firmly healed and the scars were acceptable. Slight wound dehiscence was observed in two wounds in two patients who were treated with only one cycle of wound closure. Both of these wounds healed after two weeks of dressing change. One superficial pressure ulcer was observed under the APs during wound closure, which healed after removal of the APs and conservative dressing change. For the eight wounds biopsy-positive for microorganisms before TRS therapies, six Ag dressings and two VSDs were applied: three became negative, three grew other bacteria and two continued to have the same bacterial growth. Six biopsies were positive for microorganisms at the conclusion of TRS therapies. Two were found in VSD-dressed patients, while four were found in Ag-dressed patients. Systematic symptoms of infection (fever, chills, and elevated peripheral white blood cell count) were observed in five patients. Four infections occurred after granulation tissue was resected and the wound was closed with sutures, while the other occurred after change of the VSD dressing. All of these symptomatic infections were treated successfully with antibiotic agents given according to the result of the microorganism biopsy.

Mild to moderate pain and itching were reported by 15 (36.58\%) patients; details regarding these complications are presented in Table 4.

\section{DISCUSSION}

Tissue expansion is a well-known method of repairing soft-tissue defects. The TRS is an original concept using the viscoelastic properties of the skin based on three phenomena: mechanical creep, stress relaxation and mobility of the skin (4). Mechanical creep, defined as the elongation of skin under a constant load over time beyond intrinsic extensibility, has been described as the vehicle harnessed for wound closure with presuturing, and intra- and postoperative tissue expansion. Skin-stretching devices and skin retraction with undermining (5) also have been used. The traditional implanted internal skin-tissue expander has certain disadvantages including the requirement for additional surgery for insertion of the device, extrusion, infection, the need for repeated outpatient visits to fill the expander and a long period of time before the final result is achieved. A series of other 'external skin expander' devices have been developed recently. However, due to their major drawbacks, such as secondary scars in addition to the primary defect, and wound edge necrosis induced by concentrated stress, the clinical utility of these devices is significantly limited $(6,7)$. Recently, a new device, 3M Steri-Strip S Surgical Skin Closure (3M, USA), was described by Kerrigan et al (8). This device uses noninvasive adhesion forces of the strip to ensure skin closure; hence, it is able to obviate secondary scars. The most important limitations of this method are that the adhesive loses its effectiveness due to wound exudate, and infection beneath the strips is difficult to treat, strictly limiting the clinical application of this method to clean wounds.

Results from the present single-centre cohort study demonstrated that TRS therapy is a simple effective method for closing difficult wounds with large skin and soft-tissue defects. It is relatively easy to use, does not generate donor-site morbidity, and is cosmetically acceptable when compared with skin graft and flap. All 43 wounds in the 41 patients treated with this therapy healed completely with no major complications. TRS therapy provides an additional relatively simple option for surgeons to manage such difficult defects and wounds. However, we share some observations and recommendations.

\section{Indications for application}

Theoretically, there are no absolute contraindications to TRS therapy. This method is ideal for closing complex wounds with infection or exposure of bone or internal fixation materials, or those that lack vessels suitable for flap anastomosis:

1 Stress is diverted far from the wound edge; therefore, this therapy does not disturb wound management.

2. The open drainage allowed by VSD for dressing change or vacuum drainage are conducive to treatment of wound infections.

3. TRS therapy can create better skin or soft-tissue coverage of exposed bone for further orthopedic internal fixation surgery and, by immediate primary closure of the wound, reduce the risk for acquired infection.

4. In fact, a heavily infected wound is a contraindication to a conventional internal expander and skin graft or flap transplantation (9).

5. Although the linear scar caused by the suture is inevitable, the cosmetic outcome is significantly more acceptable than that of skin graft and the donor defect in flap surgery. Large scale, randomized controlled studies with a longer follow-up period are still required to further confirm these findings.

Before deployment of a TRS device, the surgeon should be aware of several issues:

1. There are three sizes of TC: $4 \mathrm{~mm} 6 \mathrm{~mm}$ and $8 \mathrm{~mm}$. For facial and hand surgery, we used the $4 \mathrm{~mm} \mathrm{APs}$; it is possible to reduce the size of the plate by cutting it.

2. Because the adhesiveness of the APs diminishes with time due to the oils and sweat present on the skin, the skin beneath the APs must be cleaned, and the duration of this application should be limited, when using this noninvasive approach.

3. With an internal skin-tissue expander, the expander volume has been shown to have a significant influence on the failure rate (10); however, the limitations of skin expansion in different anatomical regions remains to be explored. Hence, we have no recommendation 
concerning the limits of the ratio of stretched skin and wound width when choosing this method.

4. Exposed bone and internal fixator do not constitute a contraindication to TRS therapy. They can be covered by stretched skin and soft tissue; however, local infection (including biofilm) must be effectively treated before closing the wound.

\section{Details of AP application}

We have several recommendations concerning the application of APs. 1. We do not recommended placing the APs directly on compromised damaged skin including grafted skin or skin with edema, inflammation or infection (Figure 4A).

2. The distance between the APs and the wound edge, as well as the distance between the APs, greatly affects the extent of skin stretching. Generally, the APs should be placed $2 \mathrm{~cm}$ to $5 \mathrm{~cm}$ from the wound edge, or one-half the width of the wound, depending on the bulk of the wound dressings (shorter in soft/compressible dressings, and longer in noncompressible dressings such as vacuum dressings). This placement could provide sufficient skin reserve before wound closing. The distance between two sets is related to the tension needed to approximate the wound edges and the gap between them (Figure 4B).

3. The $\mathrm{K}$-wire of the external fixator will impede skin elongation. Thus, $\mathrm{K}$-wires should be avoided when placing APs (Figure 4C).

4. Surgeons should plan to first position APs in such a way that all of the ASs will run along the designed stretching direction coinciding with the main vector of tension. The APs should first be fixed temporarily with adhesive, and then be secured and further fixed with sutures or staples. ASs should be applied and exert pull only after the APs are secured to the skin.

5. For new users, the most difficult aspect is to suture precisely across the designated pairs of oval openings on each plate. Because the plates are not transparent, it may be difficult to direct the needle tip to the desired opening in the plate. We recommend using a $3.5 \mathrm{~cm}$ diameter curved suture needle, which is most suitable for the oval opening (Figure 4D).

\section{Prevention and management of infection}

Symptomatic local infection was the most common complication during the present investigation. Our study showed that neither Ag nor VSD dressings could successfully avoid $100 \%$ of symptomatic infections. However, all of these symptomatic infections were successfully treated with antibiotic agents given according to the microorganism biopsy result. Furthermore, Ag dressing and VSD dressing can provide a closed, wet and sterile local environment. No chronic osteomyelitis was noted in any of the 30 wounds with exposed bone or internal fixation material. However, this treatment may prolong the exposure period of bone and internal fixation material (compared with primary flap surgery); hence, the risk for chronic bone infection does exist. A larger-scale study could help in answering this question. In conclusion, the key points for avoiding infections are strict aseptic technique, thorough debridement and biopsy to test for microorganisms during each invasive approach.

Mechanisms and management of dehiscence and pressure ulcer For the eight wounds in six patients without cycles of secure wound closure after wound closure, a limited extent of wound dehiscence was observed in two wounds in two patients (Figure 4E). One possible explanation is that the mean duration of the stretching cycle in the present study was $16.72 \pm 4.48$ days, which is relatively shorter than the reported duration of conventional internal skin-tissue expander. After stretching, although the skin defect was closed by suture, there are still gaps between the subcutaneous soft tissues. The retraction of subcutaneous soft tissue maybe a major cause of dehiscence (Figure 4F). Our precautions for and management of dehiscence are as follows: first, blunt dissection of the subcutaneous tissues before applying the APs, which will decrease tissue retraction, followed by application of deep

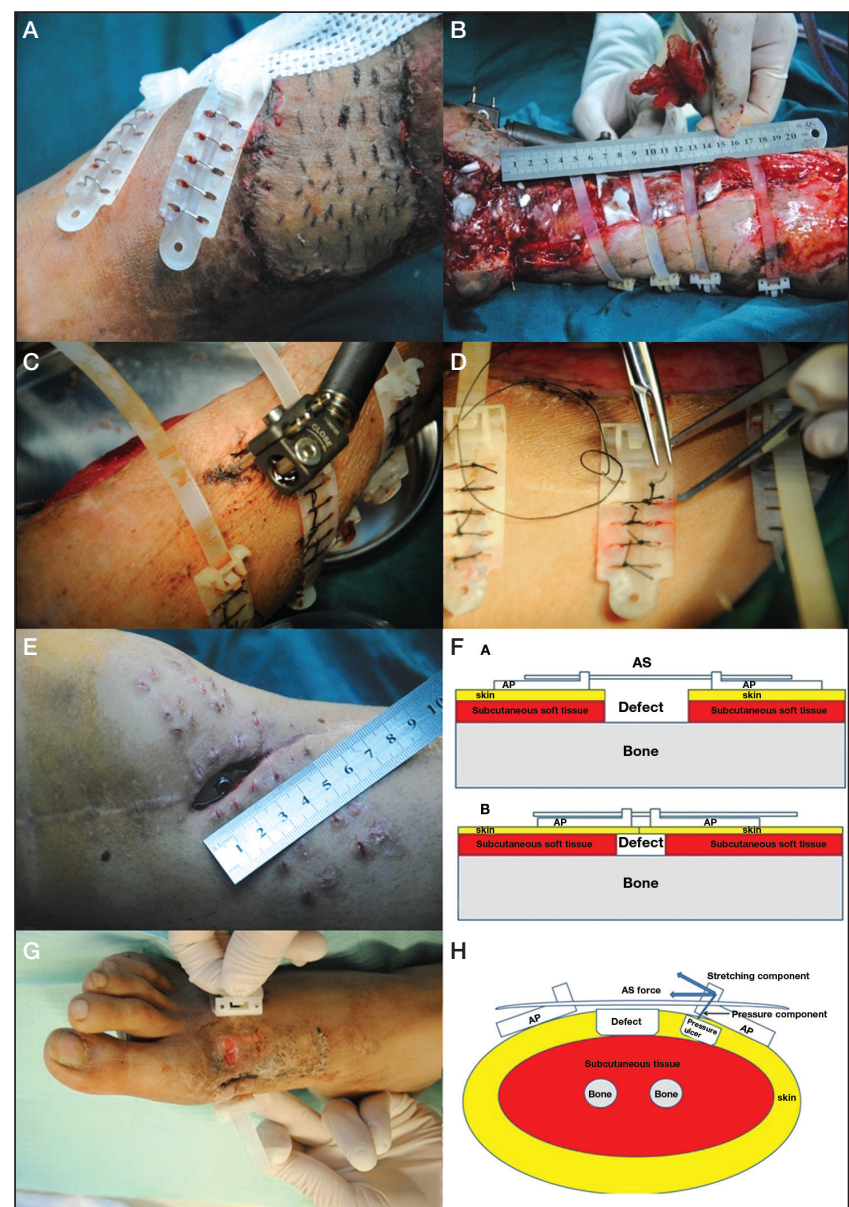

Figure 4) A Grafted skin should be carefully avoided when placing the attachment plates (APs). B Distance between two sets should relate to the tension that is needed to approximate the wound edges and the gap between them. C The K-wire of the external fixator impeded skin elongation. D We recommend using a $3.5 \mathrm{~cm}$ diameter curved suture needle because it suits the oval opening best. E Wound dehiscence after cycles closing wounds. F Simulation of the dehiscence mechanism, the retraction of subcutaneous soft tissues is the major cause of dehiscence. G Superficial pressure ulcer observed under the APs during closing wound. H Simulation of the pressure ulcer mechanism, compartment of pressure and sharp lower edge of the APs is the major cause of pressure ulcer. AS Approximation strap

sutures together with traction of the APs; and, second, after wound closure by direct suture, if the tension of the suture line is too high, a further cycle of secure wound closure is recommended. All six of the wounds that underwent further cycles healed without dehiscence. One superficial pressure ulcer was observed under the APs during wound closure (Figure 4G). This was a medial dorsal wound and the APs were located on an arc-shaped plate. The stretching forces, therefore, had a strip of pressure on the skin beneath the APs. Additionally, the lower edge of the APs was too sharp (Figure 4H). Our solution for this complication was to place a cardboard mat beneath the APs (alternatively, pressure releasing options, such as gauze or a soft sponge, are also acceptable). When the therapy cycle was completed, the APs were removed immediately, and the pressure wound was covered with a conventional dressing.

\section{Limitations}

Our study had several limitations. First, it was a single-centre, smallscale, uncontrolled study that only enrolled 41 patients with 43 wounds. Second, the wounds of the enrolled patients were very different from one another and, thus, it was difficult to evaluate and compare the scars. 
Third, although serious complications, such as osteomyelitis and wound rupture did not occur, less severe complications, such as pressure ulcer, symptomatic and asymptomatic infection, did. Hence, a larger scale, randomized cohort study, and modification of both product and technique would be required to address such problems.

\section{CONCLUSION}

The present single-centre, cohort study demonstrated that TRS therapy is a simple, effective method for closing difficult-to-heal wounds and large skin and soft-tissue defects. All of the 43 wounds we studied were completely healed. The TRS provided additional options for reconstructive surgeons to manage such defects and wounds. Symptomatic infection was the most common complication during this therapy unrelated directly to the TRS therapy. Other minor complications included dehiscence and superficial pressure ulcer. Large-scale, randomized control studies are required to investigate the effectiveness, indications, complications and cost effectiveness of TRS therapy, as well as exploring potential improvements and means of evaluating this therapy.

\section{SUMMARY}

TRS therapy is a simple effective method for closing difficult-to-heal wounds, and large skin and soft-tissue defects. However, larger-scale, randomized control studies are required to thoroughly investigate the effectiveness, indications, complications, cost effectiveness, and potential for better evaluation and improvement of TRS therapy.

\section{SUPPLEMENTARY MATERIAL}

Several additonal case presentations involving this technology are available online, go to www.pulsus.com.

ACKNOWLEDGEMENTS: The author(s) acknowledge the financial support of The National Natural Science Foundation of China (No. 81301654) and the Foundation of Education Department of Zhejiang Province (No.Y201534606 and No.Y201328265) for the research, authorship and/or publication of this article.
DISCLOSURES: The authors have no additional financial disclosures or conflicts of interests to declare.

ETHICS DECLARATION: This study was approved by the Ethics Committee of Second Affiliated Hospital, College of Medicine, Zhejiang University, China.

\section{REFERENCES}

1. Fang L, Zhou C, Yang M. Expansion concept as a new technique for expanding skin and soft tissue. Exp Ther Med 2013;6:1295-9.

2. Patel PA, Elhadi HM, Kitzmiller WJ, Billmire DA, Yakuboff KP. Tissue expander complications in the pediatric burn patient: A 10-year follow-up. Ann Plast Surg 2013.

3. Topaz M, Carmel NN, Silberman A, Li MS, Li YZ. The TopClosure(R) 3S System, for skin stretching and a secure wound closure. Eur J Plast Surg 2012;35:533-43.

4. Voinchet V, Magalon G. [Repair of skin defects by skin extension procedures]. Annales de chirurgie plastique et esthetique 1996;41:563-71.

5. Wilhelmi BJ, Blackwell SJ, Mancoll JS, Phillips LG. Creep vs. stretch: A review of the viscoelastic properties of skin. Ann Plast Surg 1998;41:215-9.

6. Fan J, Eriksson M, Nordstrom RE. External device for tissue expansion: Clinical evaluation of the skin extender. Scand J Plast Reconstr Surgery Hand Surg 1996;30:215-20.

7. Kirschke J, Georgas D, Sand M, Bechara FG. External tissue expander for closing large defects of the extremities and trunk. J Cutan Med Surg 2013;17:1-3.

8. Kerrigan CL, Homa K. Evaluation of a new wound closure device for linear surgical incisions: 3M Steri-Strip S surgical skin closure versus subcuticular closure. Plast Reconstr Surg 2010;125:186-94.

9. Austad, E. D. Contraindications and complications in tissue expansion. Facial Plast Surg 1988;5:379-82.

10. Bozkurt A, Groger A, O'Dey D, et al. Retrospective analysis of tissue expansion in reconstructive burn surgery: Evaluation of complication rates. Burns 2008;34:1113-8. 